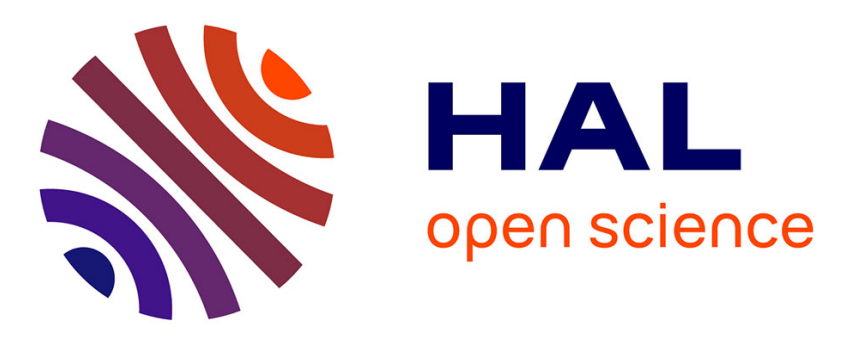

\title{
Investigating the recognition of local shapes using mid-air ultrasound haptics
}

\author{
Thomas Howard, Gerard Gallagher, Anatole Lécuyer, Claudio Pacchierotti,
} Maud Marchal

\section{- To cite this version:}

Thomas Howard, Gerard Gallagher, Anatole Lécuyer, Claudio Pacchierotti, Maud Marchal. Investigating the recognition of local shapes using mid-air ultrasound haptics. WHC 2019 - IEEE World Haptics Conference, Jul 2019, Tokyo, Japan. pp.1-6, 10.1109/WHC.2019.8816127 . hal-02121329

\section{HAL Id: hal-02121329 \\ https://hal.inria.fr/hal-02121329}

Submitted on 6 May 2019

HAL is a multi-disciplinary open access archive for the deposit and dissemination of scientific research documents, whether they are published or not. The documents may come from teaching and research institutions in France or abroad, or from public or private research centers.
L'archive ouverte pluridisciplinaire HAL, est destinée au dépôt et à la diffusion de documents scientifiques de niveau recherche, publiés ou non, émanant des établissements d'enseignement et de recherche français ou étrangers, des laboratoires publics ou privés. 


\title{
Investigating the Recognition of Local Shapes Using Mid-air Ultrasound Haptics
}

\author{
Thomas Howard ${ }^{1}$, Gerard Gallagher ${ }^{3}$, Anatole Lécuyer ${ }^{2}$, Claudio Pacchierotti ${ }^{1}$, and Maud Marchal ${ }^{3}$
}

\begin{abstract}
Mid-air haptics technologies are able to convey haptic sensations without any direct contact between the user and the haptic interface. One representative example of this technology is ultrasound haptics, which uses ultrasonic phased arrays to deliver haptic sensations. Research on ultrasound haptics is only in its beginnings, and the literature still lacks principled perception studies in this domain. This paper presents a series of human subject experiments investigating important perceptual aspects related to the rendering of $2 \mathrm{D}$ shapes by an ultrasound haptic interface (the Ultrahaptics STRATOS platform). We carried out four user studies aiming at evaluating (i) the absolute detection threshold for a static focal point rendered via amplitude modulation, (ii) the absolute detection and identification thresholds for line patterns rendered via spatiotemporal modulation, (iii) the ability to discriminate different line orientations, and (iv) the ability to perceive virtual bumps and holes. These results shed light on the rendering capabilities and limitations of this novel technology for 2D shapes.
\end{abstract}

\section{INTRODUCTION}

Mid-air haptics is an emerging group of technologies for providing haptic sensations at a distance, without any direct physical contact with the interface creating the stimuli. Several physical principles can be used to provide midair haptic stimuli: magnetism [1], acoustics [2], electric arcs [3], optics [4], and aerodynamics [5]. Among these technologies, the currently most mature one uses focused airborne ultrasound. Arrays of ultrasonic transducers produce phase-shifted acoustic waves which constructively interfere at points in space called focal points and destructively interfere elsewhere, conveying sensations by varying acoustic radiation pressure on the skin [2], [6]. The device used in this study is commercialized by Ultrahaptics and capable of creating multiple focal points whose intensity and position are updated at up to $40 \mathrm{kHz}$ [6]. Ultrahaptics technology is used in a large set of application domains, from entertainment and Virtual Reality (VR) to 3D modeling and automotive [7]. For example, Long et al. [8] presented an algorithm for rendering three-dimensional shapes. Users were shown to be able to differentiate five different shapes: a sphere, a pyramid, a horizontal prism, a vertical prism and a cube. Harrington et al. [9] and Shakeri et al. [10] demonstrated the use of ultrasound haptics in vehicular applications, showing significant reductions in visual demand, interaction times, and improved accuracy. Finally, Georgiou et al. [11] used ultrasound haptics for a VR mid-air haptic rhythm game, and Martinez et al. [12] for an immersive fantasy narrative.

This research has received funding from the EU's H2020 research and innovation programme (grant agreement No 801413, project "H-Reality").

${ }^{1}$ T. Howard and C. Pacchierotti are with Univ Rennes, IRISA, CNRS, Inria - Rennes, France.

${ }^{2}$ A. Lécuyer is with Univ Rennes, Inria, IRISA, CNRS - Rennes, France.

${ }^{3}$ G. Gallagher and M. Marchal are with Univ Rennes, INSA, IRISA, Inria, CNRS - Rennes, France.

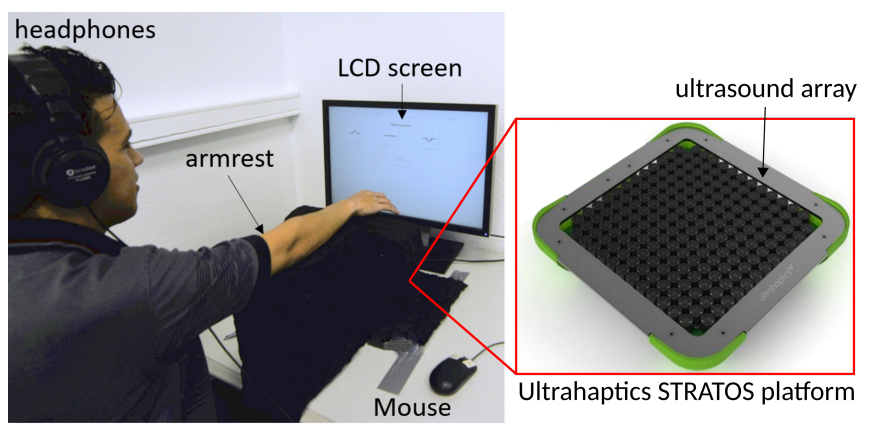

Fig. 1: Experimental setup: Participants place their dominant arm on the armrest, receiving haptic stimuli on their palm.

From a perceptual point of view, there are several studies investigating the effect of providing ultrasound haptic stimulation. Gavrilov et al. [13] perfomed pioneering work on tactile sensations evoked by ultrasound, determining detection thresholds for ultrasound stimuli from a single transducer at various frequencies. Dalecki et al. [14] expanded on this work, showing the main phenonmenon contributing to elicited tactile sensations was acoustic radiation pressure. However, to the best of our knowledge, no perception studies have been carried out using focused ultrasound phased arrays like the Ultrahaptics device.

Toward this objective, we present a series of human subjects experiments investigating the perceptual aspects associated with the rendering of $1 \mathrm{D}$ and 2D shapes, such as points, lines, bumps, and holes. We expect these results to be useful for all researchers aiming at designing new rendering techniques and immersive experiences using this technology.

\section{EXPERIMENTAL OBJECTIVE AND SETUP}

The objective of this work is to evaluate to what extent we can render $1 \mathrm{D}$ and $2 \mathrm{D}$ shapes using the Ultrahaptics technology. The following experiments measure and discuss (i) the absolute detection threshold for a static focal point rendered via amplitude modulation (Sec. [III), (ii) the absolute detection and identification thresholds for line patterns rendered via spatiotemporal modulation (Sec. IV), (iii) the ability to discriminate different line orientations (Sec. $(\mathrm{V})$, and (iv) the ability to perceive virtual bumps and holes (Sec. VI)

Experiment \#1: detecting focal points. The first experiment evaluated the absolute detection threshold for a single static focal point. For this point to be felt, the acoustic radiation pressure must vary at a given frequency, i.e., $200 \mathrm{~Hz}$ in this experiment [15]. The focal point was rendered using Amplitude Modulation (AM). When using this modality, the device generates output from every transducer such that constructive and destructive interferences at and around the focal point result in its acoustic radiation pressure oscillating 
at the desired frequency. The focal point intensity can then be varied by changing the output intensity, affecting the peak of the resulting acoustic radiation pressure. For the Ultrahaptics STRATOS platform, changes in intensity ranging between $0 \%$ and $100 \%$ correspond to peak acoustic radiation pressure at the focal point between 0-1125 $\mathrm{Pa}$.

Experiment \#2: detecting and identifying lines. Our second experiment evaluated the detection threshold for a linear pattern of a given length. For this experiment, AM is not a suitable modality, because the update rate for point positions and intensities is constrained by the chosen focal point frequency. In fact, a certain number of acoustic radiation pressure oscillation periods is required at a given position and intensity for the user to perceive the haptic stimulus. Spatiotemporal modulation (STM) was introduced by Kappus and Long [16] to address this limitation. In STM, focal points are generated with a fixed frequency (usually the maximum achievable by the device, i.e., $40 \mathrm{kHz}$ ). Since this frequency is very high, it poses significantly fewer constraints on the temporal evolution of the peak intensity and focal point position. Thus, by moving the focal point through space and/or varying the peak intensity, it is possible to achieve complex modulations of the resulting acoustic radiation pressure along 3D paths. Frier et al. [17] have also investigated the trade-off between pattern repetition rate in STM and perceived intensity. For these reasons, we used STM to display lines above the board at the optimal focal point movement speed with respect to their length $(7 \mathrm{~m} / \mathrm{s}$ in our case) while varying the intensity of the moving focal point. The experiment was performed in two parts: the first one investigated the detection threshold regardless of the perceived pattern, while the second one investigated the intensity required for users to reliably feel the displayed pattern as a line.

Experiment \#3: discriminating line orientations. Our third experiment aimed at evaluating how well users can discriminate lines oriented in four different ways. Again, we rendered line patterns on the subject's palm using STM. However, here we investigated the effect of stimulus intensity on line orientation discrimination as well as potential effects of hand movements for the same metric (passive vs. active touch).

Experiment \#4: recognizing bumps and holes. Finally, our fourth and last experiment aimed at evaluating the device's capability for displaying shapes along the z-axis, i.e., modulating the perceived local height profile. While in planes parallel to the array surface, the Ultrahaptics device can achieve a spatial resolution below $1 \mathrm{~cm}$, this is not true in planes perpendicular to the array surface. In fact, focal points tend to "stretch" along the vertical direction, with the acoustic energy spreading out over several centimeters. This is especially an issue when rendering small-scale shapes along the $\mathrm{z}$-axis. To overcome the above rendering issue, we propose to modulate the focal point intensities in a plane parallel to the device, so as to introduce the illusion of a variation of height.

Setup. The experimental setup, shown in Fig. 1, is composed of an armrest, an Ultrahaptics STRATOS platform, an LCD screen, and a pair of noise-canceling headphones. The armrest was situated $20 \mathrm{~cm}$ above the Ultrahaptics device, and was designed to keep subjects comfortable and rested throughout the duration of the experiments. Subjects were asked to sit in front of the screen, place their arm on the armrest, with their palm facing the ultrasound array and provide responses to various stimuli, depending on the experimental protocols. Subjects used a mouse to report their answers to the questions displayed on the screen. Subjects wore headphones playing pink noise throughout all experiments in order to avoid potential effects due to auditory cues arising from the device operation. Starting conditions as well as starting trial blocks were randomized between subjects so as to rule out any possible learning and fatigue effect. All subjects provided informed consent.

\section{DETECTION OF SINGLE FOCAL POINTS}

We aim at evaluating the 50\%-detection threshold for a single focal point generated using AM, both in a passive touch (static hand) and an active touch (moving hand) condition.

\section{A. Materials and Methods}

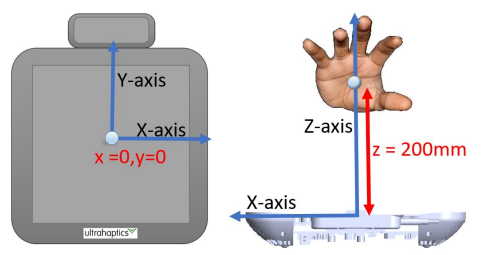

Fig. 2: A static focal point (light blue) is rendered at $20 \mathrm{~cm}$ above the centre of the board, so that the user can feel it on the palm. Only the focal point intensity is varied throughout the experiment.

Subjects were presented with a static $200 \mathrm{~Hz}$ focal point located $20 \mathrm{~cm}$ above the center of the board (see Fig. 2) for a duration of $2 \mathrm{~s}$ at different intensity levels and were asked a binary question regarding whether they felt the stimulus or not. In the passive touch condition, subjects were asked to keep their hand still while the focal point was rendered on the palm. In the active touch condition, subjects were asked to swipe their hand back and forth while following an on-screen display of a dot moving at $0.5 \mathrm{~m} / \mathrm{s}$.

We used a 1-up, 1-down staircase method to determine subjects' 50-\%detection thresholds. Trial blocks were structured so as to randomly interleave one upward staircase trial run (starting from $0 \%$ intensity) and one downward staircase trial run (starting from $100 \%$ intensity). Intensities were adjusted in $4 \%$ increments, and subject responses were recorded for each level. Each subject performed three trial blocks per condition (passive touch and active touch). Trial blocks continued until a total of 9 reversals were recorded for each of the interleaved upward and downward trial runs. The first reversal was ignored in subsequent analyses and the remaining 8 were averaged to obtain an estimate of the detection threshold, yielding two measures per trial block.

15 healthy subjects ( 8 male, 7 female; ages 22-32 (M=25); 2 left-, 13 right-handed) participated in the experiment. 


\section{B. Results and discussion}

In the passive condition, mean subject thresholds ranged from $32 \%$ to $77 \%$ (interquartile $41 \%-52 \%$ ) with a mean of $49.5 \%$ (median $46 \%$ ). In the active condition, thresholds are slightly lower, ranging from $28 \%$ to $72 \%$ (interquartile $41 \%-$ $51 \%$ ) with a mean of $48.7 \%$ (median $45.3 \%$ ). This difference between conditions is not significant (t-test). Therefore, we cannot conclude any effect of hand movement on the detection threshold for a focal point generated through AM.

\section{DETECTION AND IDENTIFICATION OF LINE PATTERNS}

We aim to investigate the 50\%-detection and 50\%identification thresholds for lines generated using STM.

\section{A. Materials and methods}
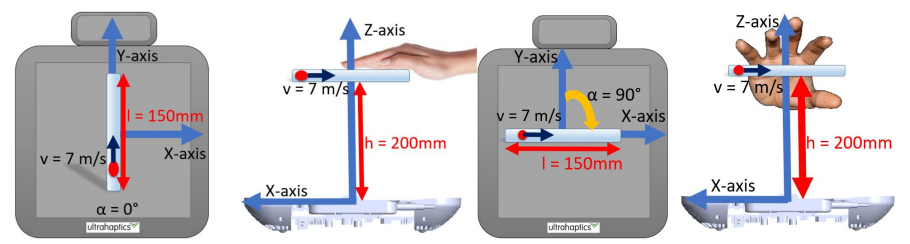

Fig. 3: Left: line pattern aligned with the device y-axis $\left(\alpha=0^{\circ}\right)$. Right: line pattern aligned with the device $\mathrm{x}$-axis $\left(\alpha=90^{\circ}\right)$. Using STM, a high-frequency focal point (red dot) is rapidly moved along a path (light blue segment). The resulting sensation is a continuous pattern in the shape of the focal point path. The perceived intensity of the pattern can be modulated by changing the focal point intensity.

Subjects were presented with 15-cm-long lines aligned with the device $\mathrm{x}$ - or $\mathrm{y}$-axis at a height of $20 \mathrm{~cm}$. We selected a focal point movement speed of $7 \mathrm{~m} / \mathrm{s}$, which is close to the best perceived intensity [17]. In both variations of the experiment, the intensities of the focal point were varied, and subjects were asked to respond to binary questions regarding either the detection or identification of the stimuli. Subjects were left free to explore the plane in which the stimuli were presented $(\mathrm{z}=20 \mathrm{~cm})$ as they saw fit.

We used 1-up, 1-down staircase methods to determine both thresholds, as we did in Sec. III. Each subject performed two trial blocks, one in a $\alpha=0^{\circ}$ and another in a $\alpha=90^{\circ}$ orientation. Subjects responded to the binary questions "Did you feel a stimulus?" and "Did you feel a line?" for the detection and identification experiments, respectively.

7 healthy subjects (5 male, 2 female, ages 22-32 (mean: 27.2), all right-handed) participated in the detection thresholds experiment, and 12 different healthy subjects ( 9 male, 3 female, ages 22-47 (mean: 28.3), 11 right handed, 1 left handed) participated in the identification thresholds experiment.

\section{B. Results and discussion}

For the detection thresholds experiment, 6 out of 7 subjects showed a lower median threshold in the $0^{\circ}$ condition when compared to the $90^{\circ}$ condition, and only 1 showed a higher median threshold in the $0^{\circ}$ condition (none of these differences were found to be significant, Wilcoxon rank-sum tests). Looking at mean subject thresholds, they ranged from $21.3 \%$ to $33.3 \%$ in the $0^{\circ}$ orientation, with a mean of $28.4 \%$ (median 29.6\%), as shown in Fig. 4. In the $90^{\circ}$ orientation, subject mean thresholds ranged from $21.8 \%$ to $36.3 \%$, with a mean (and median) of $31 \%$. The observed differences in mean thresholds between orientation were not found to be significant (Wilcoxon rank-sum test).

For the identification thresholds experiment, subject mean thresholds ranged from $23.1 \%$ to $56.8 \%$ in the $0^{\circ}$ orientation, with a mean of $42.8 \%$ (median $46 \%$ ), as shown in Fig. 4. In the $90^{\circ}$ orientation, subject mean thresholds ranged from $29.8 \%$ to $79.6 \%$, with a mean of $48.9 \%$ (median $47.8 \%$ ). These mean identification thresholds tended toward a lower median value in the $0^{\circ}$ condition, but the difference was not found to be significant (Wilcoxon rank-sum test).

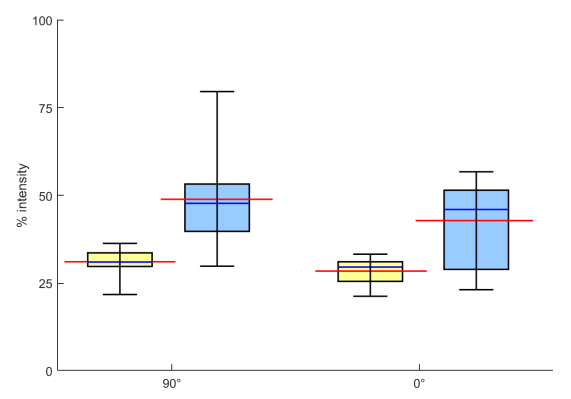

Fig. 4: Mean subject detection (yellow) and identification (blue) thresholds for lines aligned with the $\mathrm{x}$-axis (right) and $y$-axis (left). Medians are dark blue, means are in red.

For both the $0^{\circ}$ and $90^{\circ}$ orientations, the measured identification thresholds are well above the detection thresholds (median $46 \%$ for identification vs. median $29.6 \%$ for detection in $0^{\circ}$, median $47.8 \%$ for identification vs. median $31 \%$ for detection in $90^{\circ}$ ). Pairwise comparisons between these thresholds in the $0^{\circ}$ and $90^{\circ}$ orientations show these differences to be significant with $\mathrm{p}<0.05$ and $\mathrm{p}<0.01$ respectively (Wilcoxon rank-sum tests).

Both detection and identification thresholds for line patterns generated through STM were found to be lower than detection thresholds for a single point generated through AM (which is consistent with prior results from Takahashi et al. [18]). This result can be attributed to a number of factors, such as the change in modulation and possible constructive interference between successive focal points in STM, the stimulation of a larger surface area (which is consistent with previous findings on contact vibrotactile displays [19]), and even the fact that lines aligned with the y-axis allowed subjects to feel out the pattern with more sensitive parts of the hand than the palm (e.g., the fingertips).

\section{DISCRIMINATION OF LINE ORIENTATIONS}

During the previous experiment, subjects frequently reported a certain degree of confusion regarding the orientation of the line patterns, especially at lower intensities. Therefore, we decided to carry out a preliminary investigation into the capacity for discrimination of line pattern orientations at different intensity levels. 


\section{A. Materials and methods}

Subjects were presented a randomized sequence of lines with 4 different orientations, $\alpha=\left\{0^{\circ}, 45^{\circ}, 90^{\circ}, 135^{\circ}\right\}$, at 3 different intensity levels, a low-intensity level (54\%), an intermediary intensity level (77\%), and a high intensity level $(100 \%)$. The low intensity level was chosen to be above the $50 \%$-identification threshold of $90 \%$ of the sample population (see Sec. IV), ensuring that subjects almost certainly felt the pattern as a line. The intermediary intensity was chosen as the midway point between the low and maximum intensity. Subjects performed two trial blocks with 120 trials each (10 for each pair of intensity and orientation levels), one block in a passive touch condition, one in an active touch condition.

10 healthy subjects (8 male, 2 female, ages 22-44 $(\mathrm{M}=27.2)$, all right handed) participated in the study.

\section{B. Results and discussion}

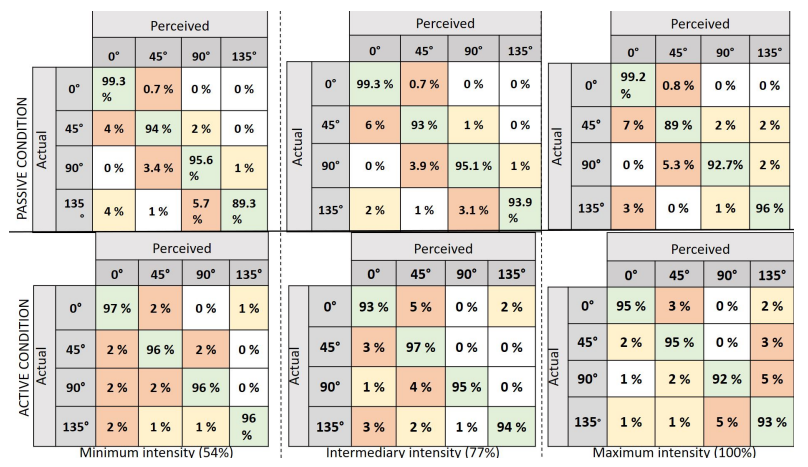

Fig. 5: Mean confusion matrices for each condition. Hit rates are highlighted in green. The most prevalent confusions are highlighted in orange, the second most prevalent in yellow.

Confusion matrices were obtained for each intensity level and averaged (see Fig. 5. Overall, the majority of orientations were correctly identified. $0^{\circ}$ lines were occasionally confused with $45^{\circ}$ or $135^{\circ}$, but never with $90^{\circ}$ lines (both in active and passive conditions). $90^{\circ}$ lines are similarly never confused with $0^{\circ}$ lines in the passive condition, but occasionally in the active condition. The most confused orientation is $135^{\circ}$ with $90^{\circ}$.

In the passive condition, $0^{\circ}$ lines are best identified, with no differences observed regarding the intensity level of the display. The $45^{\circ}$ and $135^{\circ}$ orientations seem to be the hardest to identify, showing the lowest means and largest variability in subject responses. Correct identification rates increase in mean for the $135^{\circ}$ orientation, while they tend to decrease for both the $45^{\circ}$ and $90^{\circ}$ orientations as intensity increases. Analyzing the effect on hit rates of orientation and intensity, a 2-way ANOVA shows that orientation has a significant effect on hit rates $(\mathrm{p}<0.05)$, while intensity and interactions between both do not.

In the active condition, correct identification rates do not differ much from one orientation to another. There seems to be a slight tendency toward greater variability and lower mean correct identification rates with increasing intensity, but a 2-way ANOVA shows neither orientation, nor intensity, nor interactions between both significantly affect hit rates.
When evaluating the effect of hand movement, it seems that subjects do worse on identifying $0^{\circ}$ lines in the active condition. However, overall, they show slightly higher mean correct identification rates and less variability than in the passive condition. Analysing the effect of intensity and condition (passive vs. active) on hit rates for the $0^{\circ}$ orientation, a 2-way ANOVA shows no effect of intensity, but a significant effect of condition $(\mathrm{p}<0.01)$ and no effect of interactions. 2-way ANOVAs analyzing the effects of intensity and condition on hit rates in other orientations show no significant effects.

2-way ANOVAs analyzing the effects of intensity and condition on confusion rates between pairs of orientations show no effects of intensity, condition or interactions in most cases. The confusion rate between orientations $0^{\circ}$ and $90^{\circ}$ is an exception, with a significant effect of the condition on the confusion rate $(\mathrm{p}<0.05)$. The confusion rate between orientations $90^{\circ}$ and $135^{\circ}$ is significantly affected by the interaction between intensity and condition $(\mathrm{p}<0.1)$.

\section{DISPLAYING BUMPS AND HOLES ALONG THE Z-AXIS}

We aim to investigate the identification rates of bumps and holes rendered along the device $\mathrm{z}$-axis, together with effects of orientation on shape recognition.

\section{A. Materials and methods}

Subjects were presented with a random selection of five haptic patterns (one bump, one hole, and three lines of different intensities) and asked to identify the stimulus as a line, a bump, or a hole. Each pattern was presented 30 times, i.e., the subjects felt a total of 30 bumps, 30 holes, and 10 lines for each of the 3 intensity levels. For this initial investigation, we chose to use the largest possible difference between baseline and peak intensity for both patterns, in order to maximise chances of correct identification. However, because of this, the baseline intensities for the bump and hole patterns are necessarily different. We chose to divide the line patterns into three intensity levels so as to act as potential confounders, in the case where subjects would not actually identify bumps and holes but rather differences in baseline, peak or mean intensities. Lines were designed in

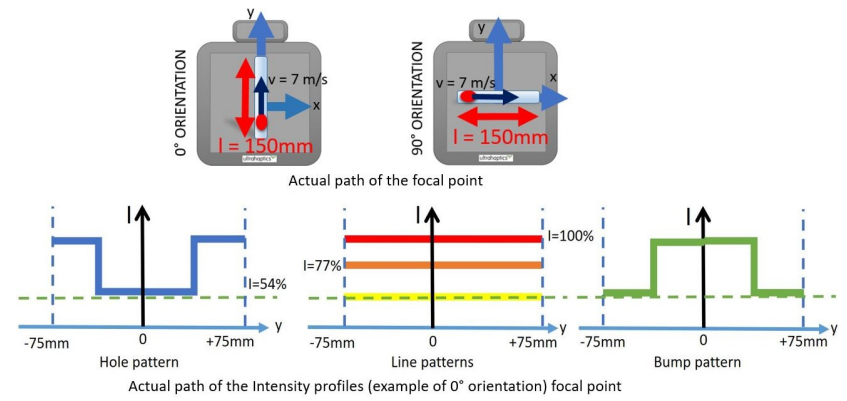

Fig. 6: Stimuli are presented in two possible orientations, $0^{\circ}$ and $90^{\circ}$ (top), with 5 different intensity profiles (bottom). Focal point intensity as a function of position is show in blue for a hole pattern; in yellow, orange and red respectively for the minimum, intermediary and maximum intensity lines; and in green for the bump pattern.

the same way as presented in Secs. IV and $\mathrm{V}$, with the three 
intensity levels selected so as to match the baseline intensities of, the hole pattern $(100 \%)$, the bump pattern $(54 \%)$ and an intermediary value $(77 \%)$, respectively. To maximize chances of identification, bumps and holes were designed with the intensity profile following a step function, with the central 1/3rd of the line at peak intensities of $100 \%$ (bump) and $54 \%$ (hole), respectively (see Fig. 6).

In addition to the recognition rate, we also investigated the effects of orientation on pattern discrimination. Subjects performed the experiment in two blocks, one with patterns aligned with the device $\mathrm{x}$ axis $\left(\alpha=90^{\circ}\right)$ and one with patterns aligned with the device y axis $\left(\alpha=0^{\circ}\right)$.

12 healthy subjects ( 8 male, 4 female; ages $23-30$ ( $M=$ $25.3)$; 10 right-handed, 2 left-handed) participated in the experiment. A questionnaire (7-point Likert scale) was filled out at the end of the experiment, rating subject confidence, fatigue, ease of the task and difficulties in identifying the different shapes.

\section{B. Results and Discussion}

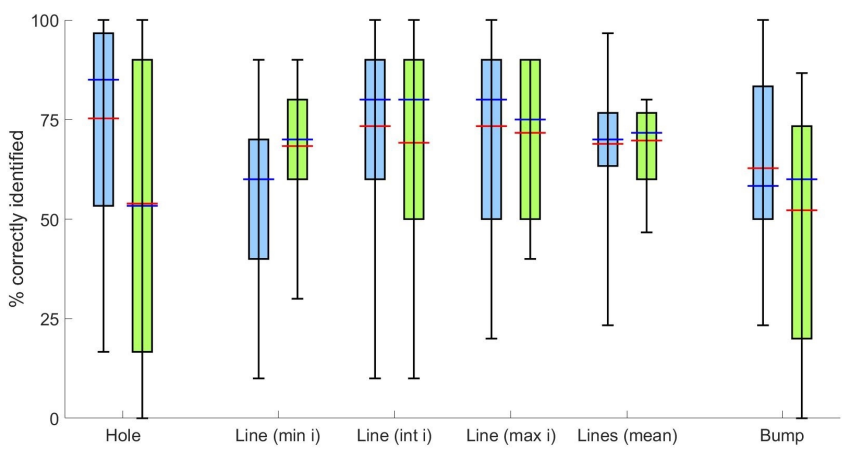

Fig. 7: Correct identification rates grouped by shape, in the $90^{\circ}$ (blue) and $0^{\circ}$ (green) conditions. Medians are shown in blue and means in red.

Correct identification rates are shown in Fig. 7. A 2way ANOVA analyzing the effects of orientation and shape on correct identification rates showed that none of these variables and their interactions have a significant effect on the identification rates.

In the $0^{\circ}$ condition, there is no difference between identification rates for lines of different intensities (69.7\% mean), and barely any difference between identification rates of bumps and holes (52.2\% and 53.9\% means respectively). Bumps and holes are identified correctly much less of the time than lines. None of the observed differences are significant (1-way ANOVA).

In the $90^{\circ}$ condition, minimum intensity lines are identified correctly less of the time $(60 \%$ mean) than intermediary or maximum intensity lines $(73.3 \%$ mean). Bumps are identified $(62.8 \%$ mean) less than holes $(75.3 \%$ mean) or intermediate and maximum lines (mean identification rate of all lines was $68.9 \%$ ), but still twice above the random chance $(33.3 \%)$. Holes are identified as much as intermediary or maximum intensity lines and above lines on average. A 1-way ANOVA shows none of the observed differences are significant. These tendencies show that it is slightly easier to correctly identify high-intensity lines, and that holes tend to be easier to identify than bumps, mirroring the subjective reports of subjects who generally expressed a greater degree of certainty when feeling holes than when feeling bumps.

In the $90^{\circ}$ condition, bumps and lines are more often confused $(24.2 \%$ of the time) than holes and lines $(11 \%)$ or bumps and holes $(9.7 \%)(\mathrm{p}<0.01$, t-test $)$. Confusion between bumps and lines is primarily due to bumps being misidentified as lines, indicating no discrimination of shape on the part of subjects (difference in medians significant with $\mathrm{p}<0.1$, Wilcoxon rank-sum test). Confusion between holes and lines seems equally due to holes being misidentified as lines, indicating no discrimination of shape, as to lines being misidentified as holes, indicating an erroneous perception of a dip in intensity along the line. Confusion between bumps and holes is mainly due to holes being misidentified as bumps, i.e., it appears that a lower intensity in the centre of a high-intensity pattern is easier to miss than a high intensity in the centre of a low intensity pattern (difference is significant with $\mathrm{p}<0.05$, t-test). Holes seem to be more often misinterpreted as lines than as bumps, confirming the idea that subjects probably "missed" the low intensity section in the centre. In the $0^{\circ}$ condition, lines and bumps are confused $25.3 \%$ of the time on average, mostly from bumps being misidentified as lines (difference in median misidentification rate significant with $\mathrm{p}<0.01$, Wilcoxon rank-sum test). Again, confusion between lines and holes (15.1\% of the time) is more or less equally distributed among lines being misidentified as holes and holes being misidentified as lines. Bumps are confused with holes $21.3 \%$ of the time, mostly from holes being misidentified as bumps. Again, this result indicates that subjects may perceive that there is an evolution in the intensity profile but do not interpret it correctly.

A detailed look at the influence of line intensity on misidentification of lines in the $90^{\circ}$ condition shows that, for minimum and intermediate intensity lines, misidentification rates are similar for bumps and holes. Maximum intensity lines are more often misidentified as bumps than holes $(\mathrm{p}<0.05$, t-test). This means that intensity at the center of the pattern is important for shape identification. In the $0^{\circ}$ condition, minimum intensity lines are mostly misidentified as bumps $(\mathrm{p}<0.1$, t-test), but more of them are identified as holes than in the $90^{\circ}$ condition. Minimum intensity lines are more often misidentified as holes than as bumps $(\mathrm{p}<0.1$, t-test), while intermediary intensity lines are equally misidentified as bumps and holes. This again tends to indicate that intensity in the centre of the pattern is determining in identification, more than intensity on the edge.

Comparisons between orientations reveal no significant differences. Subjects tended to perform similarly for both (contrary to their subjective perception indicating major preference for the $90^{\circ}$ condition). Only the identification rates for bumps and holes appear to be slightly lower, and the confusion rate between bumps and holes higher in the $0^{\circ}$ condition, which does not match subjective assessment by our subjects.

Concerning the subjective questionnaire, participants reported being quite confident (mean $M=4$, standard deviation $S D=1.6)$. They found the task quite easy $(M=3$, 
$S D=3.45)$ and were almost not fatigued $(M=2, S D=$ 2.45 ). Concerning the difficulty to perceive the different shapes, they found it moderately difficult to feel bumps $(M=3.1, S D=1)$, and slightly more difficult to feel lines $(M=4.36, S D=1.6)$ and holes $(M=4.73, S D=1.7)$. $50 \%$ of the subjects found it easier to identify bumps in the $90^{\circ}$ condition, against $25 \%$ for the $0^{\circ}$ condition and $25 \%$ which expressed no preference. Concerning identification of lines, most subjects $(83 \%)$ did not prefer a specific orientation, reflecting the almost identical identification rates for lines in both orientations. Concerning the identification of holes, $42 \%$ of subjects showed no preference while another $42 \%$ preferred the $90^{\circ}$ condition. Overall, it seems that subjects expressed greater ease of identification in the $90^{\circ}$ condition, which reflects the tendencies shown in both identification rates for bumps and holes as well as in confusion rates for all patterns. When asked about why they believed that the $90^{\circ}$ condition allowed for better identification, most subjects responded that exploring a shape along a line using the palm made things easier thanks to (i) the less differences in tactile sensitivities between the various parts of the skin being stimulated and (ii) the stimulation of a contiguous patch of skin (e.g., against fingers, that are separated by gaps).

\section{General DISCUSSION AND CONCLUSION}

This paper presented a series of user studies investigating perceptual aspects associated with the rendering of 1D and 2D shapes, such as points, lines, bumps, and holes. We determined the 50\%-detection threshold for a single focal point generated with AM, finding it to be largely independent of hand movement and on average between $48.7 \%$ and $49.5 \%$ intensity, i.e., approx. $560 \mathrm{~Pa}$ peak acoustic radiation pressure. Then, we proceeded to investigate the effects of changing the displayed pattern and modulation to gain insights about displaying lines. Using STM, we found it was possible to reduce the detection threshold to around $30 \%$ intensity, i.e., approx. $338 \mathrm{~Pa}$. However, at such low intensities, subjects did not readily identify the patterns as lines, with the threshold for a proper identification of lines being closer to $45 \%$ intensity. An extension of the current protocol to other 2D shapes with various parameters could yield further insights into the intensity required to properly feel and discriminate between them. We also performed a preliminary investigation into line orientation discrimination at various intensities, finding no significant effect of intensity or hand movement on line orientation discrimination capabilities. However, a comprehensive evaluation of angular JNDs for linear patterns and possible effects of intensity could provide insights into the best intensity setting when displaying directions. Finally, we proposed a novel solution for displaying bumps and holes by modulating the intensity profile along a linear path, and we investigated the corresponding subject discrimination abilities. While our method was found to be effective to a certain extent, an evaluation of the JND for the intensity of line patterns would be interesting both for insights into rendering of 2D shapes and for possibly enhancing the resolution obtained in our rendering method for displaying bumps and holes along the $\mathrm{z}$ axis.
In the future, we plan to study to what extent it is possible to render other 2D (and 3D) shapes with this technology, while extending the perceptual tests to other parts of the body. Combining ultrasound haptics with visual stimuli will let us study how immersive virtual environments can facilitate sensations and possibly lower thresholds. Finally, we plan to combine ultrasound haptics with other types of haptic stimuli (e.g., using wearables [20]), to extend the range of sensations provided and the range of features we can render.

\section{REFERENCES}

[1] A. Adel, M. A. Seif, G. Hölzl, M. Kranz, S. Abdennadher, and I. S. Khalil, "Rendering 3d virtual objects in mid-air using controlled magnetic fields," in Proc. IEEE/RSJ IROS, 2017, pp. 349-356.

[2] T. Iwamoto, M. Tatezono, T. Hoshi, and H. Shinoda, "Airborne ultrasound tactile display," in Proc. ACM SIGGRAPH Demos, 2008.

[3] D. Spelmezan, D. R. Sahoo, and S. Subramanian, "Sparkle: Towards haptic hover-feedback with electric arcs," in Proc. Ann. Symp. User Interface Software \& Technology, 2016, pp. 55-57.

[4] J.-H. Jun, J.-R. Park, S.-P. Kim, Y. M. Bae, J.-Y. Park, H.-S. Kim, S. Choi, S. J. Jung, S. H. Park, D.-I. Yeom et al., "Laser-induced thermoelastic effects can evoke tactile sensations," Scientific reports, vol. 5, p. 11016, 2015.

[5] S. Gupta, D. Morris, S. N. Patel, and D. Tan, "Airwave: Non-contact haptic feedback using air vortex rings," in Proc. ACM Int. Joint Conf. Pervasive and Ubiquitous Computing, 2013, pp. 419-428.

[6] T. Carter, S. Seah, B. Long, B. Drinkwater, and S. Subramanian, "Ultrahaptics: multi-point mid-air haptic feedback for touch surfaces," in Proc. ACM UIST, 2013, pp. 505-514.

[7] E. Magazine. (2019) Automotive infotainment cruises on high at ces. [Online]. Available: https://goo.gl/Nxi9iM

[8] B. Long, S. A. Seah, T. Carter, and S. Subramanian, "Rendering volumetric haptic shapes in mid-air using ultrasound," ACM Transactions on Graphics (TOG), vol. 33, no. 6, p. 181, 2014.

[9] K. Harrington, D. R. Large, G. Burnett, and O. Georgiou, "Exploring the use of mid-air ultrasonic feedback to enhance automotive user interfaces," in Proc. 10th International Conference on Automotive User Interfaces and Interactive Vehicular Applications, 2018, pp. 11-20.

[10] G. Shakeri, J. H. Williamson, and S. Brewster, "May the force be with you: Ultrasound haptic feedback for mid-air gesture interaction in cars," in Proc. 10th International Conference on Automotive User Interfaces and Interactive Vehicular Applications, 2018, pp. 1-10.

[11] O. Georgiou, C. Jeffrey, Z. Chen, B. X. Tong, S. H. Chan, B. Yang, A. Harwood, and T. Carter, "Touchless haptic feedback for VR rhythm games," in Proc. IEEE VR, 2018, pp. 553-554.

[12] J. Martinez, D. Griffiths, V. Biscione, O. Georgiou, and T. Carter, "Touchless haptic feedback for supernatural vr experiences," in Proc. IEEE VR, 2018, pp. 629-630.

[13] L. R. Gavrilov, G. V. Gersuni, O. B. Ilyinski, E. M. Tsirulnikov, and E. E. Shchekanov, "A study of reception with the use of focused ultrasound. i. effects on the skin and deep receptor structures in man," Brain Research, vol. 135, no. 2, pp. 265 - 277, 1977.

[14] D. Dalecki, S. Z. Child, C. H. Raeman, and E. L. Carstensen, "Tactile perception of ultrasound," J. Acoustical Soc. America, vol. 97, no. 5, pp. 3165-3170, 1995.

[15] K. Hasegawa and H. Shinoda, "Aerial vibrotactile display based on multiunit ultrasound phased array," IEEE Transactions on Haptics, vol. 11, no. 3, pp. 367-377, 2018.

[16] B. Kappus and B. Long, "Spatiotemporal modulation for mid-air haptic feedback from an ultrasonic phased array," J. Acoustical Soc. America, vol. 143, no. 3, pp. 1836-1836, 2018.

[17] W. Frier, D. Ablart, J. Chilles, B. Long, M. Giordano, M. Obrist, and S. Subramanian, "Using spatiotemporal modulation to draw tactile patterns in mid-air," in Proc. Int. Conf. Human Haptic Sensing \& Touch Enabled Computer Applications, 2018, pp. 270-281.

[18] R. Takahashi et al., "Lateral modulation of midair ultrasound focus for intensified vibrotactile stimuli," in Proc. Int. Conf. Human Haptic Sensing \& Touch Enabled Computer Applications, 2018, pp. 276-288.

[19] R. T. Verrillo, "Effect of contactor area on the vibrotactile threshold," J. Acoustical Soc. America, vol. 35, no. 12, pp. 1962-1966, 1963.

[20] C. Pacchierotti, S. Sinclair, M. Solazzi, A. Frisoli, V. Hayward, and D. Prattichizzo, "Wearable haptic systems for the fingertip and the hand: Taxonomy, review, and perspectives," IEEE Transactions on Haptics, vol. 10, no. 4, pp. 580-600, 2017. 